\title{
Effective Thermodynamics for a Marginal Observer
}

\author{
Matteo Polettini and Massimiliano Esposito* \\ Physics and Materials Science Research Unit, University of Luxembourg, \\ Campus Limpertsberg, 162 a avenue de la Fä̈encerie, L-1511 Luxembourg, Luxembourg
}

(Received 21 March 2017; published 13 December 2017)

\begin{abstract}
Thermodynamics is usually formulated on the presumption that the observer has complete information about the system he or she deals with: no parasitic current, exact evaluation of the forces that drive the system. For example, the acclaimed fluctuation relation (FR), relating the probability of time-forward and time-reversed trajectories, assumes that the measurable transitions suffice to characterize the process as Markovian (in our case, a continuous-time jump process). However, most often the observer only measures a marginal current. We show that he or she will nonetheless produce an effective description that does not dispense with the fundamentals of thermodynamics, including the FR and the 2nd law. Our results stand on the mathematical construction of a hidden time reversal of the dynamics, and on the physical requirement that the observed current only accounts for a single transition in the configuration space of the system. We employ a simple abstract example to illustrate our results and to discuss the feasibility of generalizations.
\end{abstract}

DOI: 10.1103/PhysRevLett.119.240601

Nonequilibrium thermodynamics is a discourse about a set of currents $\boldsymbol{J}=\left(J_{\alpha}\right)_{\alpha=1}^{n}$ flowing across an open system and about their cost, viz. the forces or affinities $\boldsymbol{F}=$ $\left(F_{\alpha}\right)_{\alpha=1}^{n}$ that need to be exerted to sustain such flows. Statistical Mechanics makes currents into random variables whose joint probability $P(\boldsymbol{J})$ is determined by the occurrence of an underlying Markovian trajectory $\omega$ in configuration space. Be they electric currents and voltages, heat flows and temperature gradients, etc., thermodynamics establishes certain universal truths, among which, in a progression that covers the 19th to 21st century time frame: (2nd law) the average steady-state rate at which entropy is delivered to the environment $\boldsymbol{F} \cdot\langle\boldsymbol{J}\rangle$ is non-negative; (FDR) near equilibrium a perturbation of the currents leads to dissipation, which is related to the current's fluctuations at equilibrium; (FR) the probability of observing positive fluctuations of the entropy production is exponentially favored if related to negative ones [1]:

$$
\frac{P(\boldsymbol{J})}{P(-\boldsymbol{J})}=\exp (t \boldsymbol{F} \cdot \boldsymbol{J}) .
$$

Notoriously, the FR holds in the long-time limit, but it can be formulated at all times upon a proper choice of the equilibrium distribution from which the initial configuration is sampled [2-4].

The above results require that all possible sources of dissipation are known. However, most often such a complete description is impossible to achieve, neither experimentally (leakages) nor theoretically (coarse-grained degrees of freedom $[5,6])$. How can then thermodynamics be established in a consistent way? In this Letter we consider a marginal observer who only monitors one current $J=J_{\alpha \equiv 1}$ flowing between two discrete configurations, and who controls a parameter that only affects the rates at which transitions between those configurations occur. We show that the observer would guess an effective affinity such that the following occurs: (i) A marginal FR for the observed current holds at large times, for any given statistical distribution of the initial state, or at all times upon a proper choice of the initial distribution (see also Refs. [7-10] for alternative formulations of a marginal FR); (ii) the effective affinity has a clear interpretation as the counteracting force needed to stall the current, i.e., to make it vanish; (iii) there exists a simple operational procedure to determine the effective affinity; (iv) the FDR at stalling derived in Ref. [11] follows as a consequence; (v) the marginal FR requires a new notion of "hidden time-reversal" (HTR), an interesting mathematical construct rich in properties, though lacking a clear operational implementation. In some sense HTR inverts the dynamics in the hidden subspace (see Ref. [12] for an analog for underdamped Langevin dynamics).

Our conclusions stand on several mathematical propositions. We only include in the Letter the outline of those proofs whose elements are necessary to interpret the results, leaving details to the Supplemental Material [13].

Story of a marginal observer.-Consider an observer who can operate a measurement apparatus that only resolves two configurations $i=1,2$. To him, the rest of the system is a black box:

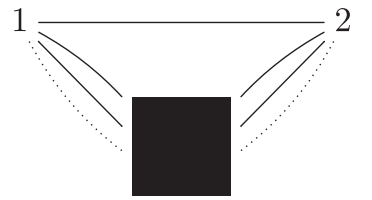

The observer controls the rate $w_{12}(x)$ at which, given that the apparatus recorded 2, the next measurement is 1 , and similarly for $w_{21}(x)$. The observer also records how often 
configuration 2 occurs with respect to 1 . This latter piece of information is quantified by the ratio $p_{1}(x) / p_{2}(x)$, where $\vec{p}(x)$ is the probability of being anywhere in configuration space, including in the black box, whose population $p_{\mathbf{.}}(x)$ is unknown. Finally, the observer handles a parameter $x$ by which he tunes the values of the rates. We assume that rates inside the black box do not depend on $x$, and that $x$ satisfies

$$
\frac{w_{12}(x)}{w_{21}(x)}=\frac{p_{1}^{\mathrm{eq}}(x)}{p_{2}^{\mathrm{eq}}(x)}=e^{x},
$$

where $\vec{p}^{\text {eq }}$ is the detailed balanced steady state when the connections to the black box are all "cut" and the edge is disconnected from the network, and as a consequence no net current is measured along 1-2. Let us give a more physical insight into the parametrization via two examples. Rates of systems in contact with heat reservoirs satisfy local detailed balance, e.g., $\log \left(w_{12} / w_{21}\right)=\left(\varepsilon_{2}-\varepsilon_{1}\right) / k_{B} T_{12}$, with $\varepsilon_{1}, \varepsilon_{2}$ energy levels and $T_{12}$ the temperature of the environmental degrees of freedom that interact with the observable transition, while other edges are at different temperatures. The parameter in this case would affect the external temperature of the reservoir affecting transition 1-2 and not the internal energy levels, which do affect other transitions. A similar example is that of a specific chemical reaction $X_{1}+C \leftrightharpoons X_{2}$ belonging to a larger network of chemical reactions, where an internal reactant $X_{1}$ is coupled to a chemostat $C$ at fixed but controllable concentration [14]. In this case, it is only possible to modify the concentration of $C$, while the internal "energy levels" are unmodifiable integer particle numbers [15].

If detailed balance is not observed and the observer measures a different value of $p_{1}(x) / p_{2}(x) \neq p_{1}^{\mathrm{eq}}(x) / p_{2}^{\mathrm{eq}}(x)$, a current $\langle J\rangle(x)=w_{12}(x) p_{2}(x)-w_{21}(x) p_{1}(x)$ flows along $1-2$. The observer then needs to formulate a minimal model that is compatible with this observation. The simplest possible setup is

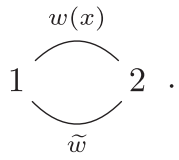

In this minimal model the black box is responsible of returning an event at 1 or 2 at some effective rates $\tilde{w}_{12}, \tilde{w}_{21}$. While this is not a viable approximation of the black-box dynamics, which is described by more advanced projection techniques [17], it is enough to replicate the average steadystate measurements of our observer.

Our first result is the determination of the effective rates. Notice that the minimal model must satisfy the global detailed balance condition obtained by lumping the transitions $w$ and $\tilde{w}$ :

$$
\frac{w_{21}(x)+\tilde{w}_{21}}{w_{12}(x)+\tilde{w}_{12}}=\frac{p_{2}(x)}{p_{1}(x)} .
$$

We will now compare this quantity with the truth-of-matter of the complete system. To illustrate our results, we employ the following example:
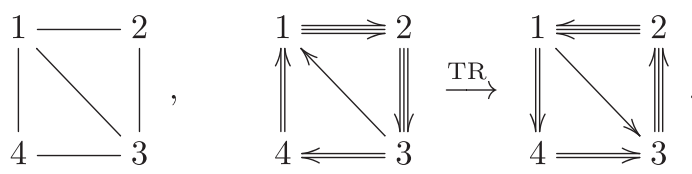

The first diagram depicts the topology, where the graph's edges connect sites (configurations). The second is a pictorial illustration of a possible steady state in configuration space, each line representing a given amount of current, obeying Kirchhoff's law of current conservation at the sites of the graph. The third is its time reversed (for later reference).

We assume that the full system evolves by the master equation $d \vec{p}(t) / d t=\mathbb{W} \vec{p}(t)$, with generator $\mathbb{W}_{i j}:=$ $w_{i j}-w_{i} \delta_{i, j}$, where rates $w_{j i}, w_{i j}>0$ are positive along all edges of the graph, and $w_{i}:=\sum_{k \neq i} w_{k i}$ are the exit rates [18]. The steady state $\vec{p}$ is the unique null vector of $\mathbb{W}$. Let $\mathbb{W}_{\left(j_{1}, \ldots, j_{n} \mid i_{1}, \ldots, i_{m}\right)}$ be the matrix obtained by removing rows $j_{1}, \ldots, j_{n}$ and columns $i_{1}, \ldots, i_{m}$. Then, Theorem 1 states that the effective rates are given by

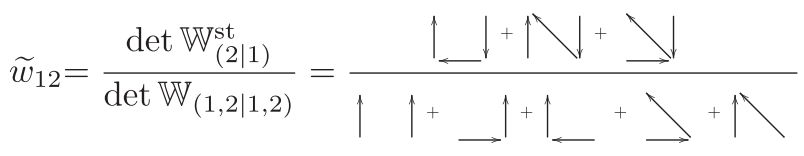

and similarly for $\tilde{w}_{21}$, where $\mathbb{W}^{\text {st }}$ is the stalling generator obtained from $\mathbb{W}$ by setting $w_{12} \equiv w_{21} \equiv 0$. Each illustrative diagram on the right-hand side represents a term of the determinant, whereby the multiplication of rates along oriented arrows is implied, e.g., $\mid$ |. Notice that, consistently with the "no- $x$-in-m" assumption, the effective rates do not depend on $x$. The proof of Theorem 1 relies on some facts in algebraic graph theory, in particular, that the steadystate probability can be written in terms of the minors of the generator [18], that by the all-minors matrix-tree theorem [19] minors can be given a graphical interpretation in terms of spanning trees, and that by the deletion-contraction principle [20] one can single out the trees of the stalling generator and the trees that pass by edge 1-2.

Effective affinity and stalling.-Supporting a steady-state current has a thermodynamic cost. According to the theory of Hill-Schnakenberg [21,22], which is the Markov-process analog of Kirchhoff's mesh analysis for electrical circuits, this cost is quantified by cycle affinities, i.e., the log-ratio of products of rates along an independent set of oriented cycles, in the two directions. In the complete system, there are a number of "real" affinities

$$
F_{\alpha}(x)=\log \prod_{i \leftarrow j \in \mathcal{C}_{\alpha}} \frac{w_{i j}}{w_{j i}} .
$$


Notice that these affinities are either in the form $F_{\alpha}(x)=$ $x-x_{\alpha}^{\text {ref }}$, with $x_{\alpha}^{\text {ref }}$ some reference value [3], if cycle $\mathcal{C}_{\alpha}$ includes the observable transition, or else do not depend on $x$. Hence, a variation of $x$ corresponds to a variation of the relevant thermodynamic forces.

In the case of the minimal model, there is only one cycle $1 \leftarrow 2 \leftleftarrows 1$ with effective affinity

$$
\tilde{F}(x):=\log \frac{w_{12}(x) \tilde{w}_{21}}{w_{21}(x) \tilde{w}_{12}}=x-x^{\mathrm{st}},
$$

where we define the stalling value of the parameter as

$$
x^{\mathrm{st}}=\log \frac{\downarrow \dashv+\uparrow \uparrow+\}{\uparrow \downarrow \uparrow+\uparrow+\downarrow}=\log \frac{p_{1}^{\text {st }}}{p_{2}^{\text {st }}} .
$$

In this latter expression we recognized $\vec{p}^{\text {st }}$ as the steady state of the stalling system where edge 1-2 is removed altogether, $\mathbb{W}^{\text {st }} \vec{p}^{\text {st }}=0$. The effective affinity then reads

$$
\tilde{F}=\log \frac{w_{12} p_{2}^{\text {st }}}{w_{21} p_{1}^{\text {st }}}
$$

and it can then be interpreted as the force that is exerted if the system is prepared in the stalling state and transition 1-2 is suddenly turned on or, conversely, as the extra force that should be applied to stall the current, as already observed by Qian [23], who dubbed it "isometric force." Such a stalling state is reached when $x=x^{\text {st }}$, where both the effective affinity and the marginal current vanish, $\langle J\rangle\left(x^{\text {st }}\right)=0$. As an important consequence, the effective affinity can be determined operationally by a simple calibration procedure, and not only based on an abstract mathematical construction. The procedure is as follows: tune $x$ to the value $x^{\text {st }}$ that makes the current vanish along the observable edge. Then $\tilde{F}=x-x^{\text {st }}$. Despite the fact that according to the local observer stalling looks like an equilibrium state, yet in the black box currents of arbitrary magnitude might be flowing [24].

Fluctuations.-So far we considered a minimal model reproducing the observer's steady-state average measurements and predicting the effective thermodynamic cost of sustaining the observable current. The question we now address is whether this marginal description is robust as it comes to fluctuations of the current.

Establishing the complete FR Eq. (1) requires a notion of time-reversed dynamics $\overline{\mathbb{W}}:=\mathbb{P} \mathbb{W}^{T} \mathbb{P}^{-1}$, where ${ }^{T}$ denotes matrix transposition and $\mathbb{P}:=\operatorname{diag}\left\{p_{i}\right\}_{i}$ is the diagonal matrix whose entries are the steady-state probabilities. Letting $\bar{P}$ be the probability measure associated to the time-reversed dynamics, under time reversal currents change sign in probability $\bar{P}(\boldsymbol{J})=P(-\boldsymbol{J})$ [see the righthand side of Eq. (6) for an illustration].

We now focus on the marginal probability of the observable current $P(J)=\int P(J) \prod_{\alpha \neq 1} d J_{\alpha}$, which, in general, does not satisfy a FR. Yet, we are able to construct a hidden time-reversal dynamics (HTR) $\tilde{W}$ such that the following marginal FR holds (Theorem 3):

$$
\frac{P(J)}{\tilde{P}(-J)}=\exp (t \tilde{F} J)
$$

where, quite remarkably, the effective affinity is precisely that estimated by the local observer.

Before introducing the HTR dynamics, let us draw some consequences. Importantly, forward and HTR dynamics differ, $P(J) \neq \tilde{P}(J)$, which leads to a substantial difference with respect to Eq. (1). However, it might be that for a "fast box," where there is a time-scale separation between observable and hidden degrees of freedom, $P(J) \approx \tilde{P}(J)$, as the case analyzed in Ref. [25] suggests. While we do derive explicit expressions for the rates of the HTR dynamics, in practice, microengineering such rates in the absence of a clear operational procedure seems to be unfeasible, so that the marginal FR might be out of experimental reach. However, standard manipulations of Eq. (12) lead to the marginal integral FR

$$
\langle\exp [-t \tilde{F}(x) J]\rangle(x)=1,
$$

which only depends on the original dynamics, while the HTR probability $\tilde{P}$ is traced out. Hence, the latter relation is experimentally accessible. Furthermore, using Jensen's inequality we obtain the effective second law $\tilde{F}(x)\langle J\rangle(x) \geq 0$; bounds on the marginal dissipation have been derived in Ref. [26]. Taking the second derivative with respect to $x$ and evaluating at stalling, we obtain the nonequilibrium FDR already proven in Ref. [11]:

$$
\left.\frac{\partial\langle J\rangle}{\partial \tilde{F}}\right|_{\mathrm{st}}=\left.\frac{\operatorname{var}^{t}(J)}{2}\right|_{\mathrm{st}} .
$$

Here, $\operatorname{var}^{t}$ is a properly time-scaled variance, considering that all cumulants of currentlike observables grow linearly in time (see Ref. [27] for details). We also used the fact that, by virtue of the parametrization, the explicit derivative $d / d x$ is equivalent to the implicit variation of the effective affinity $\partial_{\tilde{F}}$. Let us emphasize that this is not just a technical subtlety: it underlies the possibility of interpreting our results in operational terms.

Hidden time reversal.-We now specify the HTR dynamics, by defining the operator 


$$
\begin{aligned}
\tilde{\mathbb{W}}: & =\mathbb{W}-\mathbb{W}_{\mathrm{st}}+\overline{\mathbb{W}}_{\mathrm{st}} \\
& =\left(\begin{array}{cccc}
-w_{1} & w_{12} & w_{31} \frac{p_{1}^{\mathrm{st}}}{p_{3}^{\mathrm{s}}} & w_{41} \frac{p_{1}^{\mathrm{st}}}{p_{4}^{\mathrm{st}}} \\
w_{21} & -w_{2} & w_{32} \frac{p_{2}^{\mathrm{st}}}{p_{3}^{\mathrm{st}}} & 0 \\
w_{13} \frac{p_{3}^{\mathrm{st}}}{p_{1}^{\mathrm{st}}} & w_{23} \frac{p_{3}^{\mathrm{st}}}{p_{2}^{\mathrm{st}}} & -w_{3} & w_{43} \frac{p_{3}^{\mathrm{st}}}{p_{4}^{\mathrm{st}}} \\
w_{14} \frac{p_{4}^{\mathrm{st}}}{p_{1}^{\mathrm{st}}} & 0 & w_{34} \frac{p_{4}^{\mathrm{st}}}{p_{3}^{\mathrm{st}}} & -w_{4}
\end{array}\right),
\end{aligned}
$$

where $\overline{\mathbb{W}}_{\mathrm{st}}=\mathbb{P}_{\mathrm{st}} \mathbb{W}_{\mathrm{st}}^{T} \mathbb{P}_{\mathrm{st}}^{-1}$, and $\mathbb{P}_{\mathrm{st}}$ is the diagonal matrix with entries given by the components of the stalling steady state. Crucially, $\tilde{\mathbb{W}}$ can be proven to be a Markov jumpprocess generator (Theorem 2). Exit rates (diagonal elements) are the same as in the original dynamics $\mathbb{W}$. Furthermore, the upper-left $2 \times 2$ block (the only nonvanishing block of $\mathbb{W}-\mathbb{W}_{\text {st }}$ ) is unchanged while the rest of the generator undergoes time reversal, with respect to its proper stalling steady state. So, in a way, the HTR is the best attempt to invert the dynamics everywhere but in the observable subsystem, whence its name. The following diagram is a pictorial illustration of HTR of the steady currents:

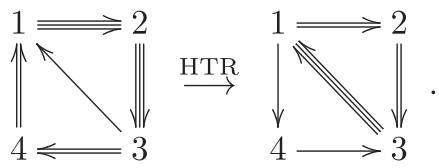

Notice that, in this example, the cycle currents' direction is inverted in the black box and preserved along the observable edge, but their intensities cannot be exactly preserved as this would violate Kirchhoff's law of current conservation at the sites. As regards Kirchhoff's loop law, we can show (Theorem 5) that the HTR has affinities $-F_{\alpha}$ along all cycles that do not include $1-2$ and affinity $2 \tilde{F}-F_{\alpha}$ for all cycles that do include 1-2. Therefore, at a stalling steady state where $\tilde{F}=0$ one has exact inversion of all the affinities and currents, and $\tilde{\mathbb{W}}\left(x^{\mathrm{st}}\right)=\overline{\mathrm{W}}\left(x^{\mathrm{st}}\right)$, which we call the condition of marginal detailed balance.

The proof of Theorem 3 relies on a direct comparison of the path probabilities [28] $P(\omega)$ and $\tilde{P}(\omega)$ of the original and the HTR dynamics, and by taking appropriate marginals. Another, more elegant way to derive the same result is via the generating function of the (time-) scaled current's cumulants (SCGF) $\lambda(q)=\langle J\rangle q+\frac{1}{2} \operatorname{var}(J) q^{2}+\cdots \quad$ in terms of which, after a bilateral Laplace transform, the marginal FR Eq. (12) takes the form of a marginal Lebowitz-Spohn symmetry [29]

$$
\tilde{\lambda}(q)=\lambda(\tilde{F}-q) .
$$

Let us outline the proof of this latter fact. To discuss possible generalizations, we allow for a current supported on several edges, $J=\sum_{i j} \varphi_{i j} J_{i j}$, with $\varphi_{i j}=-\varphi_{j i}$. It is well known that the SCGF is the Perron-Froebenius eigenvalue of the operator $\mathbb{M}(q)$ obtained by replacing offdiagonal entries of $\mathbb{W}$ by $w_{i j} e^{-\varphi_{i j} q}$, while keeping the diagonal exit rates. Then, letting $\mathbb{R}(q)$ be the diagonal matrix with entries the right eigenvector of $\mathbb{M}(q)$, one can construct an auxiliary Markovian generator $\mathbb{W}(q)=$ $\mathbb{R}(q) \mathbb{M}(q)^{T} \mathbb{R}(q)^{-1}-\lambda(q) \rrbracket$ with the property that a given rare fluctuation of the current according to dynamics $\mathbb{W}=$ $\mathbb{W}(0)$ is the typical current according to $\mathbb{W}(q)$, for some value of $q$ [30]. We show in Theorem 4 and corollaries that, for a single edge current, at $q=\tilde{F}$ the SCGF vanishes, $\lambda(\tilde{F})=0$, the HTR coincides with the auxiliary dynamics $\tilde{\mathbb{W}}=\mathbb{W}(\tilde{F})$, and the right eigenvector has a physical interpretation as the stalling steady state $\mathbb{R}(\tilde{F})=\mathbb{P}_{\text {st }}$. Equation (17) follows from matrix similarity $\square$.

This formalism allows us to significantly improve on our results. We can go back to the issue of the long-time limit and relax the assumption showing that if the observer prepares the system in the stalling state $\vec{p}^{\text {st }}$ by tuning $x$ to $x^{\text {st }}$, and then suddenly turns it back on, the FR holds at all times (see Theorem 4).

In Fig. 1 we plot the SCGF for the original and the HTR dynamics, and for their symmetric obtained by $q \rightarrow \tilde{F}-q$. The plot clearly shows that the marginal FR holds. The latest argument we outlined might suggest that our results could generalize to the case where several transitions

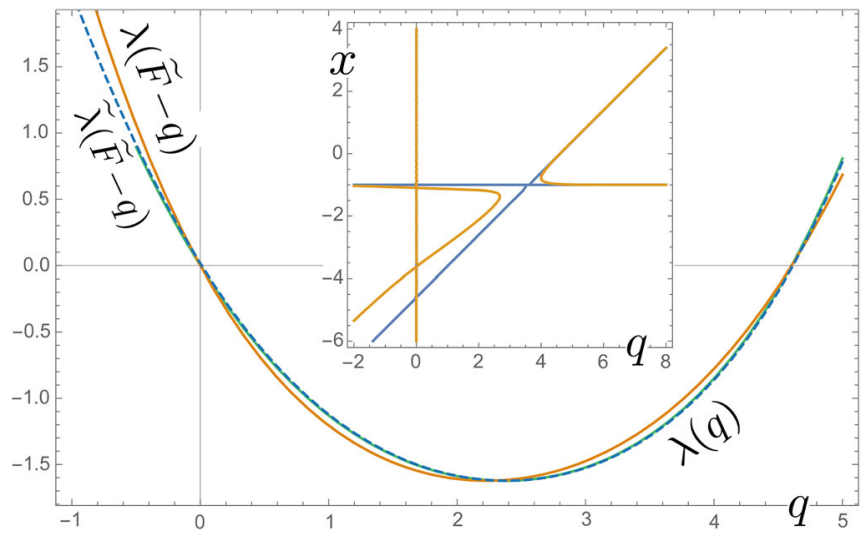

FIG. 1. We consider the model in Eq. (6) with rates $1=w_{31}=$ $w_{14}=w_{32}=w_{43}=w_{21} /(1+x)$ and $10=w_{13}=w_{41}=w_{23}=$ $w_{34} e^{-y}=w_{12} e^{-x} /(1+x)$. Main frame: For $x=y=0$, we plot the SCGF $\lambda(q)$ and its symmetric $\lambda(\tilde{F}-q)$; the two do not coincide and only meet at $0, \tilde{F} / 2$, and $\tilde{F}$, showing that the FR does not hold. We also plot (dashed) $\tilde{\lambda}(\tilde{F}-q)$, which perfectly coincides with $\lambda(q)$, showing that the FR is restored by the hidden-TR dynamics. Inset: When only transition $1-2$ is counted, for $y=0$, straight lines are the loci where $\lambda(q, x) \equiv 0$; apart from the trivial $q=0$ and $x=-1$ lines, the diagonal line corresponds to the effective affinity $\tilde{F}(x)$, showing that it is linear in $x$. The curvy lines are obtained when we also count 34 [by setting $\varphi_{i j}=\delta_{i, 1} \delta_{j, 2}+\delta_{i, 3} \delta_{j, 4}-(i \leftrightarrow j)$ and $\left.y=x\right]$. The effective affinity is no longer linear in $x$. 
contribute to the observable current; mathematically, all is needed is that there exists another value $q=\tilde{F}$ at which $\lambda(\tilde{F})=0$. However, in our previous physical construction it was crucial that the effective affinity was linear in the parameter $x$, and that $\mathbb{R}(\tilde{F})$ could be interpreted as the stalling steady state. This is not the case in general, as shown in the incept in Fig. 1. Hence, one must be prudent in claiming generality.

The case of currents supported on several edges, where symmetries enter the picture [31], is cogent: a complete treatment is in preparation. However, we can turn the above considerations upside down: if marginal fluctuation and response relations do not work, then there is a nontrivial hidden interaction between the observable and hidden degrees of freedom.

This project could not possibly come to light without thorough discussions trough the years with Bernhard Altaner. The research was supported by the National Research Fund Luxembourg (Project No. FNR/A11/02) and by the European Research Council, project NanoThermo (ERC-2015-CoG Agreement No. 681456).

*matteo.polettini@uni.lu

[1] D. Andrieux and P. Gaspard, J. Stat. Phys. 127, 107 (2007).

[2] G. Bulnes-Cuetara, M. Esposito, and A. Imparato, Phys. Rev. E 89, 052119 (2014).

[3] M. Polettini and M. Esposito, J. Stat. Mech. (2014) P10033.

[4] U. Seifert, Phys. Rev. Lett. 95, 040602 (2005).

[5] M. Esposito, Phys. Rev. E 85, 041125 (2012).

[6] S. Bo and A. Celani, J. Stat. Phys. 154, 1325 (2014).

[7] D. Hartich, A. C. Barato, and U. Seifert, J. Stat. Mech. (2014) P02016.

[8] N. Shiraishi and T. Sagawa, Phys. Rev. E 91, 012130 (2015).

[9] M. L. Rosinberg and J. M. Horowitz, Europhys. Lett. 116, 10007 (2016).

[10] G. E. Crooks and S. Still, arXiv:1611.04628.
[11] B. Altaner, M. Polettini, and M. Esposito, Phys. Rev. Lett. 117, 180601 (2016).

[12] H. Qian, Phys. Lett. A 378, 609 (2014).

[13] See Supplemental Material at http://link.aps.org/ supplemental/10.1103/PhysRevLett.119.240601 for proofs of the theorems.

[14] M. Polettini and M. Esposito, J. Chem. Phys. 141, 024117 (2014).

[15] However, as regards our theory, the case of chemical reaction networks is significantly more complicated because currents are defined along several edges, and because the topology of the chemical network affects its thermodynamics [16].

[16] M. Polettini, A. Wachtel, and M. Esposito, J. Chem. Phys. 143, 184103 (2015).

[17] B. Bravi and P. Sollich, Phys. Biol. 14, 045010 (2017).

[18] B. Gaveau and L. S. Schulman, J. Math. Phys. (N.Y.) 37, 3897 (1996).

[19] S. Chaiken, SIAM J. Algebr. Discrete Methods 3, 319 (1982).

[20] A. D. Sokal, Surveys Combinatorics 327, 173 (2005).

[21] J. Schnakenberg, Rev. Mod. Phys. 48, 571 (1976).

[22] T. L. Hill, J. Theor. Biol. 10, 442 (1966).

[23] H. Qian, Biophys. Chem. 67, 263 (1997).

[24] A. W. C. Lau, D. Lacoste, and K. Mallick, Phys. Rev. Lett. 99, 158102 (2007); D. Lacoste, A. W. C. Lau, and K. Mallick, Phys. Rev. E 78, 011915 (2008).

[25] G. Bulnes Cuetara, M. Esposito, G. Schaller, and P. Gaspard, Phys. Rev. B 88, 115134 (2013).

[26] G. Bisker, M. Polettini, T. R. Gingrich, and J. M. Horowitz, J. Stat. Mech. (2017) 093210.

[27] A. Wachtel, J. Vollmer, and B. Altaner, Phys. Rev. E 92, 042132 (2015).

[28] M. F. Weber and E. Frey, Rep. Prog. Phys. 80, 046601 (2017).

[29] J. L. Lebowitz and H. Spohn, J. Stat. Phys. 95, 333 (1999).

[30] R. Chetrite and H. Touchette, Ann. Henri Poincaré 16, 2005 (2015).

[31] M. Polettini, G. Bulnes-Cuetara, and M. Esposito, Phys. Rev. E 94, 052117 (2016). 